\title{
Importance of Lattice Constants in QM/MM Calculations on Metal- Organic Frameworks
}

\author{
Bo Yang ${ }^{1, *}$, Xin-Ping $\mathrm{Wu}^{2}$, Laura Gagliardi ${ }^{3, *}$ and Donald G. Truhlar ${ }^{1, *}$
}

${ }^{1}$ Department of Chemistry, Inorganometallic Catalyst Design Center, Chemical Theory Center, and Minnesota Supercomputing Institute, University of Minnesota, Minneapolis, Minnesota 55455, United States.

${ }^{2}$ Key Laboratory for Advanced Materials and Joint International Research Laboratory for Precision Chemistry and Molecular Engineering, Feringa Nobel Prize Scientist Joint Research Center, Centre for Computational Chemistry and Research Institute of Industrial Catalysis, School of Chemistry and Molecular Engineering, East China University of Science and Technology, 130 Meilong Road, Shanghai, 200237, P.R. China.

${ }^{3}$ Department of Chemistry, Pritzker School of Molecular Engineering, James Franck Institute, Chicago Center for Theoretical Chemistry, The University of Chicago, 5735 S Ellis Ave., Chicago, IL 60637, United States

\footnotetext{
*E-mail: b.yang3227@gmail.com

*E-mail: lgagliardi@uchicago.edu

*E-mail: truhlar@umn.edu
}

\section{TABLE OF CONTENTS}

1. Cluster model calculations using Gaussian $16 \quad$ S-2

2. Spin states of Ir-based complexes $\quad$ S-2

3. MM parameters $\quad$ S-3

References 
1. Cluster model calculations using Gaussian 16

Relative energies $\left(\Delta E_{\text {full-om }}\right)$, as defined by Eq. 2 of the main text, were computed for organometallic complexes $\mathbf{A}\left((\right.$ phen $\left.) \operatorname{Ir}(\text { Bpin })_{3}\right), \mathbf{B}\left(\right.$ eq-(phen) $\left.\operatorname{Ir}(\mathrm{Bpin})_{2} \mathrm{Cl}\right)$, and $\mathbf{C}$ (axi-(phen) $\left.\operatorname{Ir}(\mathrm{Bpin})_{2} \mathrm{Cl}\right)$ using the Gaussian $16^{1}$ program. We used the M06-L density functional ${ }^{2}$ with the def2-TZVP basis set ${ }^{3,4}$ for Ir, N, B, and $\mathrm{O}$ atoms, and the def2-SVP4 basis set for $\mathrm{C}$ and $\mathrm{H}$ atoms; effective core potentials were used to treat core electrons of the Ir atom. Results are summarized in Scheme S1. Comparing to the relative energies computed using the CP2K program $^{5}$ (Scheme 2 of main text), we see that the Gaussian 16 calculations give slightly higher relative energies with $\Delta E_{\text {full-QM }}(\mathbf{B})$ increased by $4.9 \mathrm{kcal} / \mathrm{mol}$ and $\Delta E_{\text {full-Qм }}(\mathbf{C})$ increased by $3.9 \mathrm{kcal} / \mathrm{mol}$.

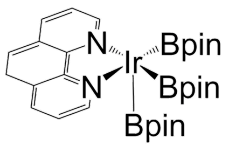

A

0.0

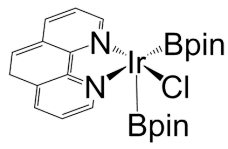

B

7.3

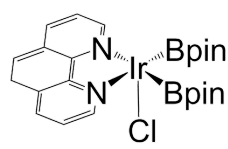

C

38.6

Scheme S1. Organometallic complexes A, B, and $\mathbf{C}$ with their relative energies $\left(\Delta \mathrm{E}_{\text {full-QM, }} \mathrm{kcal} / \mathrm{mol}\right)$ calculated using the Gaussian 16 program.

\section{Spin states of Ir-based complexes}

Using the Gaussian 16 program and the method discussed above, we computed the singlet, triplet, and quintet spin states for complex $\mathbf{A}$ in which the Ir atom has a formal oxidation state of +3 . Our results show that complex A prefers the singlet spin state; the triplet and quintet spin states are respectively 25.3 and $91.1 \mathrm{kcal} / \mathrm{mol}$ higher in energy than the singlet state. Since these energy differences are quite large and since the Ir atoms in complexes B, C, and other Ir-containing complexes studied in this paper also have a formal oxidation state of +3 , only the singlet spin state is considered for these complexes. 
The MM parameters we used for the present calculations are the ones in the original BTW paper. ${ }^{6}$

In order to run QM/MM calculations by the scheme of eq 1, one needs MM parameters for the entire system even though parameters used only for the QM region do not affect the final results because the MM interactions in the QM region cancel out. This requires some parameters that are not present in the original force field, even though they do not affect the final results. For completeness, we here give the additional parameters optimized for the QM region with the inclusion of the IR complexes:

\begin{tabular}{|c|c|c|c|c|c|c|c|c|}
\hline \multirow{4}{*}{$\begin{array}{l}\text { atom } \\
\text { atom } \\
\text { atom } \\
\text { atom }\end{array}$} & 400 & \multirow{2}{*}{$\begin{array}{l}\mathrm{Du} \\
\mathrm{H}\end{array}$} & \multicolumn{3}{|c|}{ a "dummy atom" } & \multicolumn{3}{|c|}{$\begin{array}{ll}0 & 0.0000\end{array}$} \\
\hline & 997 & & \multicolumn{3}{|c|}{ "Hlink" } & 1.0080 & \multirow[t]{2}{*}{1} & \\
\hline & 998 & $\mathrm{C}$ & \multicolumn{3}{|c|}{ "C-C3-benzene" } & $6 \quad 12$. & & 3 \\
\hline & 999 & Ir & "Ir' & & 77 & 192.2000 & 7 & \\
\hline $\mathrm{vdw}$ & 997 & & 1.62 & & 200 & 0.923 & & \\
\hline bond & 902 & 11 & & $001.3 ?$ & & & & \\
\hline bond & 999 & 12 & & $00 \quad 2.2$ & & & & \\
\hline bond & 902 & 997 & & 0001.0 & & & & \\
\hline bond & 999 & 26 & $5 .($ & 002.02 & 2555 & & & \\
\hline bond & 999 & 5 & 5.0 & 001.61 & 342 & & & \\
\hline bond & 999 & 1 & 5.0 & $00 \quad 2.30$ & 742 & & & \\
\hline bond & 9 & 912 & 5.0 & $00 \quad 1.34$ & 115 & & & \\
\hline bond & 912 & 998 & & $000 \quad 1.4$ & 182 & & & \\
\hline bond & 913 & 5 & 5.0 & $00 \quad 1.10$ & & & & \\
\hline bond & 998 & 998 & & $000 \quad 1.4$ & 2378 & & & \\
\hline bond5 & 999 & 9 & & 002.18 & 8732 & & & \\
\hline bond5 & 9 & 998 & & 001.3 & 7064 & & & \\
\hline bond5 & 26 & 6 & 5.0 & $00 \quad 1.40$ & 427 & & & \\
\hline bond5 & 998 & 399 & & .0001 .4 & 42378 & & & \\
\hline angle & 26 & 1 & 26 & 2.000 & 104.0 & 000 & & \\
\hline angle & 9 & 999 & 12 & 2.000 & 120.0 & 000 & & \\
\hline angle & 26 & 999 & 12 & 2.000 & 120 & 0000 & & \\
\hline angle & 9 & 999 & 26 & 2.000 & 119.8 & 3935 & & \\
\hline angle & 9 & 999 & 5 & 2.000 & 129.2 & 825 & & \\
\hline angle & 9 & 999 & 1 & 2.000 & 86.06 & 530 & & \\
\hline angle & 5 & 999 & 5 & 2.000 & 90.00 & 00 & & \\
\hline angle & 26 & 999 & 26 & 2.000 & 85. & 1593 & & \\
\hline angle & 26 & 999 & 5 & 2.000 & 70.4 & 073 & & \\
\hline angle & 26 & 999 & 1 & 2.000 & 105.5 & 5987 & & \\
\hline angle & 5 & 999 & 1 & 2.000 & 109.8 & 890 & & \\
\hline angle & 999 & 9 & 912 & 2.000 & 127. & 6555 & & \\
\hline angle & 999 & 26 & 6 & 2.000 & 125.0 & 853 & & \\
\hline angle & 9 & 912 & 915 & 2.000 & 116. & 2450 & & \\
\hline angle & 9 & 912 & 902 & 2.000 & 122. & 8580 & & \\
\hline angle & 915 & 912 & 998 & 2.000 & 121 & 1.1475 & & \\
\hline angle & 998 & 912 & 902 & 2.000 & $11 \mathrm{~S}$ & .5150 & & \\
\hline angle & 912 & 912 & 915 & 2.000 & & 1.0350 & & \\
\hline angle & 912 & 912 & 998 & 2.000 & & .7305 & & \\
\hline angle & 170 & 913 & 5 & 2.000 & 116. & 6563 & & \\
\hline
\end{tabular}




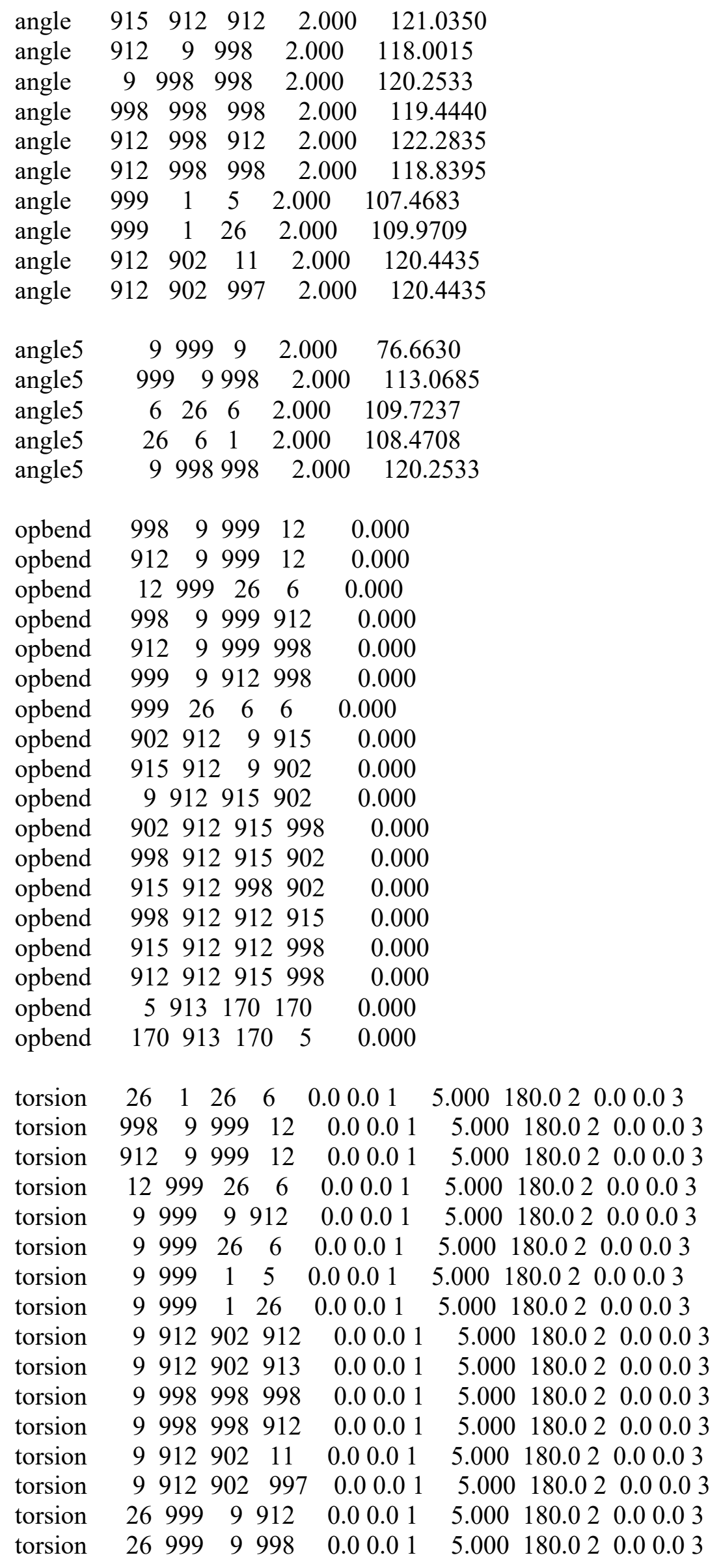




\begin{tabular}{|c|c|c|c|c|}
\hline tors & 26999 & 266 & 0.00 .01 & $\begin{array}{llllll}5.000 & 180.02 & 0.0 & 0.03\end{array}$ \\
\hline torsion & 26999 & 1 & 0.00 .01 & $5.000 \quad 180.02 \quad 0.00 .03$ \\
\hline ion & 26999 & 126 & 0.00 .01 & $\begin{array}{llllll}5.000 & 180.0 & 0.0 & 0.03\end{array}$ \\
\hline ion & 266 & $\begin{array}{lll}1 & 1 & 0\end{array}$ & 0.00 .01 & $5.000 \quad 180.02 \quad 0.0 \quad 0.03$ \\
\hline sion & 5999 & 9912 & 0.00 .01 & $\begin{array}{llllll}5.000 & 180.02 & 0.0 & 0.03\end{array}$ \\
\hline ion & 5999 & 9998 & 0.00 .01 & $\begin{array}{llllll}5.000 & 180.0 & 0.0 & 0.03\end{array}$ \\
\hline ion & 5999 & 266 & 0.00 .01 & $\begin{array}{lllll}5.000 & 180.02 & 0.0 & 0.03\end{array}$ \\
\hline ion & 5999 & 15 & 0.00 .01 & $\begin{array}{llllll}5.000 & 180.02 & 0.0 & 0.03\end{array}$ \\
\hline sion & 5999 & 126 & 0.00 .01 & $\begin{array}{lllll}5.000 & 180.02 & 0.0 & 0.03\end{array}$ \\
\hline ion & 5913 & 170192 & 0.00 .01 & $\begin{array}{llllll}5.000 & 180.02 & 0.0 & 0.03\end{array}$ \\
\hline rsion & 1999 & 9912 & 0.00 .01 & $\begin{array}{llllll}5.000 & 180.02 & 0.0 & 0.03\end{array}$ \\
\hline sion & 1999 & 9998 & 0.00 .01 & $\begin{array}{llllll}5.000 & 180.02 & 0.0 & 0.03\end{array}$ \\
\hline on & 1999 & 266 & 0.00 .01 & $\begin{array}{llllll}5.000 & 180.02 & 0.0 & 0.03\end{array}$ \\
\hline rsion & 126 & $\begin{array}{lll}6 & 1 & 0\end{array}$ & $0.00 .01 \quad 5$ & $5.000 \quad 180.02 \quad 0.00 .03$ \\
\hline ion & 9999 & 912915 & 0.00 .01 & $\begin{array}{lllll}5.000 & 180.02 & 0.0 & 0.03\end{array}$ \\
\hline ion & 9999 & 912902 & 0.00 .01 & $\begin{array}{llllll}5.000 & 180.02 & 0.0 & 0.03\end{array}$ \\
\hline ion & 9999 & 998998 & 0.00 .01 & $\begin{array}{llllll}5.000 & 180.02 & 0.0 & 0.03\end{array}$ \\
\hline n & 99926 & $6 \quad 1$ & 0.00 .01 & $\begin{array}{lllll}5.000 & 180.02 & 0.0 & 0.03\end{array}$ \\
\hline ion & $999 \quad 1$ & 26 & 0.00 .01 & $5.000 \quad 180.02 \quad 0.00 .03$ \\
\hline ion & 9989 & 912915 & 0.00 .01 & $\begin{array}{lllll}5.000 & 180.02 & 0.0 & 0.03\end{array}$ \\
\hline ion & 9989 & 912902 & 0.00 .01 & $\begin{array}{llllll}5.000 & 180.02 & 0.0 & 0.03\end{array}$ \\
\hline ion & 998912 & 2902912 & 0.00 .01 & $\begin{array}{llllll}5.000 & 180.02 & 0.0 & 0.03\end{array}$ \\
\hline ion & 998912 & 2902913 & 0.00 .01 & $\begin{array}{llllll}5.000 & 180.02 & 0.0 & 0.03\end{array}$ \\
\hline ion & 998912 & 912915 & 0.00 .01 & $\begin{array}{lllll}5.000 & 180.02 & 0.0 & 0.03\end{array}$ \\
\hline ion & 998912 & 2912998 & 0.00 .01 & $\begin{array}{lllll}5.000 & 180.02 & 0.0 & 0.03\end{array}$ \\
\hline ion & 998998 & 3998998 & 0.00 .01 & $\begin{array}{llllll}5.000 & 180.02 & 0.0 & 0.03\end{array}$ \\
\hline ion & 998998 & 998912 & 0.00 .01 & $\begin{array}{lllll}5.000 & 180.02 & 0.0 & 0.03\end{array}$ \\
\hline ion & 998912 & $2902 \quad 11$ & 0.00 .01 & $\begin{array}{llllll}5.000 & 180.02 & 0.0 & 0.03\end{array}$ \\
\hline ion & 998912 & 902997 & 0.00 .01 & $\begin{array}{lllll}5.000 & 180.02 & 0.0 & 0.03\end{array}$ \\
\hline ion & 9129 & 998998 & 0.00 .01 & $\begin{array}{llllll}5.000 & 180.02 & 0.0 & 0.03\end{array}$ \\
\hline ion & 912912 & 2998912 & 0.00 .01 & $5.000 \quad 180.020 .00 .03$ \\
\hline ion & 912912 & 998998 & 0.00 .01 & $\begin{array}{lllll}5.000 & 180.02 & 0.0 & 0.03\end{array}$ \\
\hline ion & 915912 & 2998912 & 0.00 .01 & $5.000 \quad 180.020 .00 .03$ \\
\hline ion & 915912 & 2998998 & 0.00 .01 & $5.000 \quad 180.020 .00 .03$ \\
\hline ion & 915912 & 2912915 & 0.00 .01 & $\begin{array}{llllll}5.000 & 180.0 & 2 & 0.0 & 0.03\end{array}$ \\
\hline ion & 915912 & 2912998 & 0.00 .01 & $5.000 \quad 180.020 .00 .03$ \\
\hline ion & 915912 & $2902 \quad 11$ & 0.00 .01 & $\begin{array}{llllll}5.000 & 180.02 & 0.0 & 0.03\end{array}$ \\
\hline ion & 915912 & 902997 & 0.00 .01 & $\begin{array}{lllll}5.000 & 180.02 & 0.0 & 0.03\end{array}$ \\
\hline ion & 902912 & 2998912 & 0.00 .01 & $\begin{array}{lllll}5.000 & 180.02 & 0.0 & 0.03\end{array}$ \\
\hline ion & 902912 & 2998998 & 0.00 .01 & $\begin{array}{llllll}5.000 & 180.02 & 0.0 & 0.03\end{array}$ \\
\hline ion5 & 9999 & 9998 & 0.00 .01 & $\begin{array}{llllll}5.000 & 180.0 & 0.0 & 0.03\end{array}$ \\
\hline ion5 & 9998 & 9989 & 0.00 .01 & $\begin{array}{lllll}5.000 & 180.02 & 0.0 & 0.03\end{array}$ \\
\hline torsions & $999 \quad 9$ & 998998 & 0.00 .01 & $\begin{array}{llllll}5.000 & 180.02 & 0.0 & 0.03\end{array}$ \\
\hline torsion5 & 626 & $6 \quad 1$ & 0.00 .01 & $\begin{array}{lllll}5.000 & 180.02 & 0.0 & 0.03\end{array}$ \\
\hline torcion & 266 & 11 & 0.00 .01 & $5.000 \quad 180.020 .00 .03$ \\
\hline
\end{tabular}


(1) Frisch, M. J.; Trucks, G. W.; Schlegel, H. B.; Scuseria, G. E.; Robb, M. A.; Cheeseman, J. R.; Scalmani, G.; Barone, V.; Petersson, G. A.; Nakatsuji, H.; Li, X.; Caricato, M.; Marenich, A. V.; Bloino, J.; Janesko, B. G.; Gomperts, R.; Mennucci, B.; Hratchian, H. P.; Ortiz, J. V.; Izmaylov, A. F.; Sonnenberg, J. L.; Williams-Young, D.; Ding, F.; Lipparini, F.; Egidi, F.; Goings, J.; Peng, B.; Petrone, A.; Henderson, T.; Ranasinghe, D.; Zakrzewski, V. G.; Gao, J.; Rega, N.; Zheng, G.; Liang, W.; Hada, M.; Ehara, M.;

Toyota, K.; Fukuda, R.; Hasegawa, J.; Ishida, M.; Nakajima, T.; Honda, Y.; Kitao, O.; Nakai, H.; Vreven, T.; Throssell, K.; Montgomery, J. A., Jr.; Peralta, J. E.; Ogliaro, F.; Bearpark, M. J.; Heyd, J. J.; Brothers, E. N.; Kudin, K. N.; Staroverov, V. N.; Keith, T. A.; Kobayashi, R.; Normand, J.; Raghavachari, K.;

Rendell, A. P.; Burant, J. C.; Iyengar, S. S.; Tomasi, J.; Cossi, M.; Millam, J. M.; Klene, M.; Adamo, C.;

Cammi, R.; Ochterski, J. W.; Martin, R. L.; Morokuma, K.; Farkas, O.; Foresman, J. B.; Fox, D. J.

Gaussian 16 (Revision A.03), Gaussian, Inc.: Wallingford, CT, 2016.

(2) Zhao, Y.; Truhlar, D. G. J. Chem. Phys. 2006, 125, 194101-194101.

(3) Weigend, F. Phys. Chem. Chem. Phys. 2006, 8, 1057-1065.

(4) Weigend, F.; Ahlrichs, R. Phys. Chem. Chem. Phys. 2005, 7, 3297-3305.

(5) Hutter, J.; Iannuzzi, M.; Schiffmann, F.; VandeVondele, J. Rev. Comput. Mol. Sci. 2014, 4, 15-25.

(6) Bristow, J. K.; Tiana, D.; Walsh, A. J. Chem. Theory Compt. 2014, 10, 4644-4652. 